\title{
La competencia trabajo en equipo y liderazgo en el desarrollo de Proyectos Final de Carrera en la ETSIE
}

\section{Cortés Meseguer ${ }^{a}$, N. Lajara de Camilleri ${ }^{b}$ y A. Rovira ${ }^{c}$}

${ }^{a}$ Dpto. de Construcciones Arquitectónicas. Universitat Politècnica de València luicorme@upv.es, ${ }^{b}$ Dpto. de Economía y Ciencias Sociales. CEGEA. Universitat Politècnica de València, nalade@cegea.upv.es y ${ }^{\mathrm{c}} \mathrm{CIIM}$. Dpto. de Ingeniería Mecánica y de Materiales. Universitat Politècnica de València, arovira@mcm.upv.es.

\begin{abstract}
The development of the Final Projects in the Universitat Politècnica de València is focused on being done by only one student and, increasingly, with greater time constraints due to the adaptability of degrees and tis limits in credits. Even in a professional level, the students will take part in a teamwork and, furthermore, they will be group leaders.

In the School of Engineering in Construction (ETSIE) it is being developed a working system for carrying Final Projects related with the subject "Intervention in Historic Buildings". In this group, although the presentation of the work is individual, they should help each other in different phases of the project, as it is virtually impossible to perform various activities by a single student. In this way, it is developed the teamwork and leadership competency by each of the students who make up the group, because in the end and largely the result will depend as much on their personal involvement and on the interaction among the members of the team.
\end{abstract}

Keywords: transversal competencies, teamwork and leadership, Final Project. 
La competencia trabajo en equipo y liderazgo en el desarrollo de Proyectos Final de Carrera en la ETSIE

\begin{abstract}
Resumen
El desarrollo de los Proyectos Final de Carrera de la Universitat Politècnica de València está enfocado a que se realicen por un único alumno y, cada vez más, con mayores restricciones temporales debido a las adaptaciones de los grados y sus limitaciones en créditos. Sin embargo, a nivel profesional los ahora estudiantes formarán parte de un equipo de trabajo e, incluso, acutarán como líder.

En la Escuela Técnica Superior de Ingeniería en la Edificación (ETSIE) se está desarrollando una forma de trabajo para la realización de Proyectos Final de Carrera relacionados con la asignatura "Intervenciones en Edificaciones Históricas". En dicho grupo y aunque la presentación de los trabajos es individual, deben ayudarse mutuamente en distintas fases del proyecto, ya que es prácticamente imposible el poder realizar distintas actividades por un único estudiante. De esta forma se desarrolla la competencia de trabajo en equipo y liderazgo por cada uno de los estudiantes que forman el grupo, porque al final y en gran medida, el resultado va a depender tanto de su implicación personal como de las interacciones entre los miembros del equipo.
\end{abstract}

Palabras clave: competencias transversales, trabajo en equipo y liderazgo, Proyecto Final de Carrera.

\title{
1. Introducción
}

Con la evolución proceso de enseñanza -aprendizaje, el sistema educativo debe abrirse a nuevas tendencias o nuevos enfoques, sobre todo, a las nuevas demandas del mercado profesional. Esta comunicación presenta una experiencia llevada a cabo por profesores de la asignatura "Intervenciones en Edificaciones Históricas", los cuales han ofertado Proyectos Final de Carrera (PFC) relacionados con la temática de la asignatura. Estos PFC están basados en intervenciones sobre edificaciones reales y la realización de estos proyectos supera el alcance del trabajo que pueda realizar un estudiante. Por tanto, se han formado grupos de trabajo de formados por varios estudiantes, con el objeto de abordar distintos aspectos de la restauración de los eficicios. La interrelación entre los distintos PFC aparece porque se deben actuar sobre sistemas de una misma edificación. Por tanto, aunque se traten distintos elementos del edificio, no sólo deben respectar la

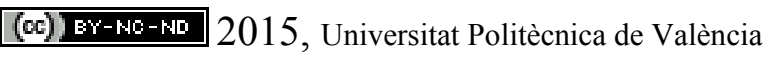


estructura y estética del edificio, sino que las distintas actuaciones deben ser compatibles entre sí.

\section{Objetivos}

En este trabajo se presenta la experiencia llevada a cabo, cuyos objetivos principales son:

- Fomentar la competencia de trabajo en equipo en la realización del PFC, aunque finalmente la autoría del informe sea única.

- Trabajar la competencia de liderazgo en equipos de trabajo con el fin de lograr los objetivos de cada uno de los miembros del equipo.

\section{Desarrollo de la innovación}

La presente comunicación establece los condicionantes que se desarrollan en un grupo de PFC en la modalidad de "ntervenciones en Edificaciones Históricas". Si bien cada alumno tiene un trabajo distinto a sus compañeros y la entrega y presentación de los mismos es en formato individual, debido a la limitación personal en la realización de distintas tareas concretas durante el desarrollo del proyecto, deben contar con la ayuda de algún compañero del grupo. Estas limitaciones quedan definidas por la limitación de tiempo, la limitación humana a la hora de realizar distintas tareas, como por ejemplo, la toma de mediciones en edificios y, sobre todo, la intelectual. La experiencia piloto se realizó con un grupo de 10 alumnos.

No solo se puede ni se produce esa ayuda en determinados momentos, sino que puede ser una ayuda continuada. Es en este momento, cuando se relaciona el trabajo en equipo y liderazgo y se forma una relación de reciprocidad entre el individuo y el grupo, ya que los alumnos quieren aspirar a la obtención de la máxima calificación posible en la evaluación y saben que su aportación personal en el grupo va a influir cualitativamente en otro compañero. 
La competencia trabajo en equipo y liderazgo en el desarrollo de Proyectos Final de Carrera en la ETSIE

El trabajo aborda las fases en las que es necesaria esa aportación personal en otros compañeros, mostrando los resultados obtenidos, en algunos casos inesperados. No solo se realiza una ayuda puntual por parte de otros compañeros, sino que en la mayoría de casos, es una ayuda continuada llegándose a convertir en un grupo de trabajo, ya que las correcciones también son grupales y cada alumno observa y puede analizar lo que ha realizado el compañero. Al realizarse las correcciones de los distintos trabajos a modo de tutorías grupales, el conocimiento adquirido y la visión crítica de cada uno de los participantes incrementa considerablemente.

Aunque se trabajan también otras competencias transversales, lo que atañe a la presente comunicación es la de trabajo en equipo y liderazgo, resaltando de esta experiencia que el liderazgo viene a mostrarse cuando se está realizando el trabajo de campo (toma de datos y levantamiento de la edificación histórica), ya que el alumno "titular" del proyecto es el que debe realizar las indicaciones oportunas para obtener todos los datos que este necesita y realizar un trabajo óptimo. Ni que decir, que según el carácter y disposición del alumno ayudante, se producirá una interacción de ambos miembros y que con el trabajo grupal que se está realizando, se está realizando un aprendizaje común a la hora de abordar el estudio de una edificación histórica.

\section{Resultados}

A nivel de trabajo en la competencia de trabajo en equipo y liderazgo, de los 10 alumnos que formaron parte en el método de trabajo, no hubo ninguna queja por parte de ningún miembro, sino que hubo más complicidad, si cabe, entre ellos y aumentando la competencia e influyendo en una mayor ambición en los trabajos.

A nivel académico, los resultados obtenidos son, en primer lugar, una mayor experiencia de los alumnos a la hora de abordar los estudios en edificios históricos, ya que a parte de su propia experiencia personal en el trabajo individual, hay que sumar la visión que están adquiriendo de los trabajos de

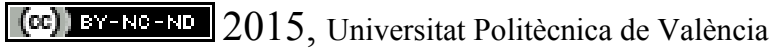


otros compañeros, ya que cada uno puede desarrollar ciertos aspectos particulares.

Por otro lado, gracias al seguimiento continuo, los alumnos son conocedores de la evolución de su trabajo individual y también del resto del grupo, debiendo ser capaces de analizar críticamente el estado de los mismos en cada una de las fases.

Por último, y a excepción de dos alumnos, los resultados académicos fueron excelentes. De los 10 alumnos, 8 trabajaron en grupo y obtuvieron las calificaciones de 8 (Notable), cinco 9 (Sobresaliente), un 10 (Sobresaliente) y un 10 (Sobresaliente Matrícula de Honor). Los dos alumnos que abandonaron el grupo obtuvieron un 6 (Bien).

\section{Conclusiones}

Es curioso, el alto grado de motivación e implicación que pueden llegar a presentar los alumnos, salvo contadas excepciones, ya que el conocido PFC es la parte de sus estudios más acorde a su futuro profesional.

También hay que destacar el grado de complicidad que puede llegar a existir entre el grupo y el profesor tutor, ya que al ser un aprendizaje guiado pero no preestablecido, refuerza ese vínculo humano.

Los resultados confirman que el trabajo en equipo ha contribuido a mejorar la calificación obtenida en el PFC.

Finalmente, es necesario remarcar el alto grado de implicación que debe presentar el profesor/tutor, ya que requiere de un esfuerzo en la preparación y seguimiento de las sesiones, que las grupales solían ser de cuatro horas continuadas un día a la semana, durante un cuatrimestre.

\section{Referencias}

Capítulo de un libro

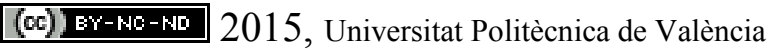


La competencia trabajo en equipo y liderazgo en el desarrollo de Proyectos Final de Carrera en la ETSIE

BLANCO, A. y ALBA, E. (2009). "La participación de los estudiantes y el trabajo en equipo" en Desarrollo y evaluación de competencias en Educación Superior. Madrid : Narcea de Ediciones.

\section{Ponencias de congresos}

BOLUMAR, C., ROVIRA, A., TORRES, R. PEREZ DE LOS COBOS, M. (2013). "La inclusión de prácticas externas en una asignatura como instrumento de la formación de futuros profesionales". En INNODOCT. Valencia: Editorial Universitat Politècnica de València.

LAJARA-CAMILLERI, N., CORTES MESEGUER, L. BAÑON-GOMIS, A.J., TRINIDAD, A. (2014). "Propuestas de experiencias docentes en el desarrollo y evaluación de la competencia "trabajo en equipo y liderazgo"' en INNODOCT, International Conference on Innovation, Documentation and Teaching Tech. Valencia.

LILlO GINER, S., PEREZ DE LOS COBOS CASINELlO, M., RODRIGO MOLINA, A., ANDRES FERRER, J., CORTES MESEGUER, L. (2013). "La evaluación entre iguales en el marco de la tutoría grupal, estrategia docente para la mejora del proceso de enseñanza-aprendizaje en alumnos de primeros cursos" en XX1 CUIEET. Valencia: Ed. Escuela Técnica Superior de Ingeniería del Diseño.

POBLETE, M. (1998). "Evaluación del Clima de Equipos de Trabajo" En IV Congreso Nacional de Psicología del Trabajo y de las Organizaciones. Valladolid. 